\title{
Sleep in Patients With Schizophrenia
}

\author{
Fabio Ferrarelli ${ }^{1}$
}

Published online: 11 April 2015

(C) Springer International Publishing AG 2015

\begin{abstract}
Numerous electrophysiological and neuroimaging studies have reported neurophysiological and cognitive deficits in schizophrenia patients during wakefulness. However, these findings have been inconsistently replicated across different groups of patients, thus complicating the identification of underlying neuronal defects. Sleep minimizes possible waking-related confounds, including decreased motivation and presence of active symptoms. Additionally, the two main sleep rhythms, slow waves and spindles, reflect the intrinsic activity of cortico-thalamic circuits and are associated with cognitive activities, including learning and memory, occurring during wakefulness. In this review, I will present the most relevant sleep findings in schizophrenia, with particular emphasis on several recent studies that have consistently reported sleep spindle deficits in patients with schizophrenia. I will then elaborate on how these findings may contribute to a better understanding of the neurobiology of schizophrenia as well as to the development of novel pharmacological and nonpharmacological interventions to ameliorate the symptoms and cognitive impairments of schizophrenia patients.
\end{abstract}

Keywords Sleep high-density EEG $\cdot$ Slow waves $\cdot$ Sleep spindles · Thalamo-cortical circuits · Schizophrenia ·

Plasticity $\cdot$ Cognitive impairments

This article is part of the Topical Collection on Sleep and Psychological Disorders

Fabio Ferrarelli

fferrarelli@uwhealth.org

1 University of Wisconsin-Madison, Madison, WI, USA

\section{Introduction}

Schizophrenia is a severe mental disorder that has significant, long-lasting negative effects on the patients as well as the entire society [1]. The term schizophrenia was introduced by Bleuler over a hundred year ago to describe the breaking up or splitting of psychic functioning he observed in these patients [2], and the diagnosis of this disorder is still based exclusively on clinical symptoms. However, a symptom-based approach has not increased our understanding of schizophrenia, given that the occurrence and severity of clinical symptoms vary across the course of the illness, and these symptoms are often shared across psychiatric diagnoses [3]. Over the past three decades, schizophrenia researchers have begun measuring cognitive and neurophysiological parameters in healthy subjects and schizophrenia patients, based on the assumption that deficits in these parameters reveal dysfunctions in neuronal mechanisms that are stable over time and largely symptom-independent [4]. Several neurophysiological [5] as well as cognitive [6] impairments have been reported during wakefulness in patients with schizophrenia; nonetheless, these findings have been inconsistently replicated across different groups of patients, thus complicating the identification of underlying neuronal defects.

Sleep offers several advantages for investigating dysfunctions in brain circuits in patient with schizophrenia. Sleep represents an ideal resting state, wherein waking-related confounding factors, including fluctuation in attention, decreased cognitive ability, and presence of active symptoms are minimized. Furthermore, the two main non-rapid eye movement (non-REM) sleep rhythms, slow waves and spindles, reflect the activity of complementary thalamo-cortical circuits. Slow 
waves are $1-\mathrm{Hz}$ neuronal oscillations characterized by large amplitude, positive-negative deflections, which are generated by cortical neurons and propagated by cortico-cortical and cortico-thalamo-cortical circuits [7]. Sleep spindles are waxing and waning, $11-16 \mathrm{~Hz}$ oscillations, which are initiated by the thalamic reticular nucleus and regulated by thalamothalamic and thalamo-cortical circuits [8]. Slow waves and sleep spindles have also been shown to implement sleepspecific plastic changes related to daytime cognitive activities, including learning and memory, in both healthy subjects $[9$, $10]$ and patients with schizophrenia [11, 12•]. Thus, reduced slow wave and/or spindle parameters may contribute to the identification of defects in specific brain circuits in schizophrenia, which are implicated in the pathophysiology of this disorder and underlie some of the symptoms and cognitive deficits commonly experienced by schizophrenia patients.

In this article, I will present the most relevant sleep findings in patients with schizophrenia, which were obtained primarily using electrophysiological recordings. I will then focus on several recent studies that have consistently reported sleep spindle deficits in schizophrenia, including first break and chronic patients, as well as in first-degree relatives of schizophrenia probands. Finally, I will review the possible neuronal and molecular mechanisms underlying sleep spindle deficits in schizophrenia, and discuss how pharmacological as well as non-pharmacological interventions reverting spindle deficits may improve some of the clinical symptoms and cognitive deficits of schizophrenia patients.

\section{Sleep Findings in Patients With Schizophrenia}

\section{Sleep Architecture}

Sleep disturbances have been consistently observed in patients with schizophrenia [13]. Sleep abnormalities often precede the onset of psychotic symptoms in first break individuals and can predict an acute decompensation in chronic, remitted patients with schizophrenia [14]. Sleep impairments have been also observed in prodromal individuals [15] and are often associated with worse cognitive function [16], one of the most persistent and treatment-refractory deficits observed in schizophrenia [17]. Numerous studies have investigated changes in the sleep architecture of schizophrenia patients. The most commonly reported abnormalities include a longer sleep latency, increased waking after sleep onset, as well as reduced sleep efficiency [18]. A reduction in deep NREM sleep, or SWS, has been reported in different groups of schizophrenia, including medication naive [19] and unmedicated [20] patients, but overall SWS deficits have been inconsistently found and appears to affect only $50 \%$ of psychotic patients [21].

\section{Slow Wave Activity (1-4 Hz)}

Only a handful of studies have investigated changes in sleep EEG activity, and especially in slow waves, in patients with schizophrenia. In two initial studies, Keshavan et al. found reductions in the delta $(1-4 \mathrm{~Hz})$ as well as theta $(4-8 \mathrm{~Hz})$ frequency bands in a subset $(N=19)$ of thirty schizophrenia patients in relation to comparison subjects [20] whereas Hiatt et al. reported a decrease in delta frequency activity in 10-min segments from the middle of non-REM sleep periods in five unmedicated schizophrenia patients [22]. However, a number of more recent studies failed to establish any difference in slow wave power $[23,24]$ as well as several slow oscillation parameters [25•,26 $]$ between patients with schizophrenia and healthy comparisons. Those inconsistent findings about reduced slow wave activity (SWA) in schizophrenia confirm previous reports that slow wave deficits may involve just a subgroup of those patients. Consistent with this assumption, lower SWA has been more often reported in institutionalized patients with profound cognitive impairment [18] as well as in schizophrenia patients with prominent negative symptoms [27].

\section{Sleep Spindles (11-16 Hz)}

Regarding sleep spindle findings in schizophrenia, Hiatt et al. reported higher spindle density during the first NREM sleep episode in five schizophrenia patients relative to healthy subjects [22], whereas two other studies performing sleep EEG recordings in nine [28] and 11 [19] schizophrenia patients and normal controls found no difference in spindle parameters between groups. Of note, these data were collected on a fairly small number of patients as well as employing a limited number of channels. By contrast, more recent work from our group employing am high density (hd)-EEG system ( $N=256$ channels) showed marked deficits in sleep spindle activity in schizophrenia patients compared to healthy as well as psychiatric controls. In an initial study, we recorded eighteen patients with schizophrenia, seventeen healthy controls, as well as fifteen patients with depression and found a marked decrease in EEG spindle power as well as in several sleep spindle parameters, including amplitude, duration, and number in schizophrenics compared to subjects from the other two groups during the first NREM sleep cycle [25•]. Sleep spindles were detected using an in-house algorithm and a fourth parameter, integrated spindle activity (ISA) which was calculated by integrating the absolute amplitude of each detected spindle divided by total non-REM sleep duration had the largest effect size. Specifically, ISA values did not overlap for 16 of 18 schizophrenia patients when compared to both healthy comparison and depression subjects. In a follow-up, whole night sleep hd-EEG study on forty-nine schizophrenia patients, forty-four healthy subjects, and twenty non-schizophrenic 
psychiatric patients on antipsychotic medications, we confirmed spindles deficits in a larger group of schizophrenics and established that these deficits were present throughout the night [26•]. Furthermore, we found no difference in the spindle activity of the other two groups, thus suggesting that spindle deficits are unlikely to be related to antipsychotics and are possibly specific for schizophrenia patients. We also investigated slow wave power and several slow wave parameters, including incidence, amplitude, down and up slopes and found no difference between schizophrenics and both healthy and psychiatric controls (Fig. 1). Altogether, these findings suggest that sleep spindle deficits may be implicated in the neurobiology of schizophrenia [29]. In an attempt to assess whether spindle impairments are present at illness onset as well as in family members of schizophrenia probands, Manoach et al. compared sleep spindle activity in antipsychotic-naïve individuals newly diagnosed with psychosis, young non-psychotic first-degree relatives of schizophrenia patients, as well as two samples of healthy controls matched to the patients and their relatives [30॰]. They found that first-break schizophrenia patients had significantly reduced spindle activity compared to both healthy controls and early course patients with other psychotic disorders. They also established that relatives of schizophrenia probands had reduced spindle activity compared with controls. Furthermore, the authors examined the relations of spindle parameters with cognitive measures and symptom ratings and found that reduced spindle activity correlated with impairment in cognitive executive functions as well as with higher level of positive symptoms in first break patients with schizophrenia [30॰]. Whereas more studies on larger groups of patients are needed to establish the extent and consistency of spindle deficits in schizophrenia, these findings from different research groups suggest that spindle abnormalities precede the onset of acute psychotic symptoms, persist throughout the course of the illness, and may account for some of the cognitive deficits and symptoms commonly experienced from schizophrenia patients.

\section{Neuronal and Molecular Mechanisms Underlying Sleep Spindle Deficits in Schizophrenia}

Sleep spindles are generated and modulated within the thalamo-cortical system [31]. Specifically, the thalamic reticular nucleus (TRN) is considered the spindle pacemaker, based on the absence of spindles in the thalamo-cortical systems after disconnection from the TRN as well as the presence of spindle rhythmicity in the isolated reticular neurons, whereas cortical inputs are thought to contribute to initiate and amplify sleep spindle oscillations [32]. All recent studies reporting spindle activity impairments in schizophrenia found that the incidence was the most defective spindle parameter in schizophrenia patients. Spindle incidence also correlated with the clinical symptoms and impaired cognitive performances in those patients. Moreover, one of these studies established that schizophrenia patients had no deficits in cortically generated slow waves compared to healthy controls, thus suggesting that dysfunction in a TRN/thalamus circuit may be primarily responsible for sleep spindle deficits in schizophrenia [26•]. The TRN has also been implicated in blocking, or selectively enhancing, the transmission of peripheral stimuli to the cortex through sensory gating and attentional modulation, respectively. Intracranial recordings in primates have shown that the regulation of visual attention involves higher activity in
Fig. 1 Schizophrenia patients have reduced sleep spindles, but not slow waves compared to healthy controls

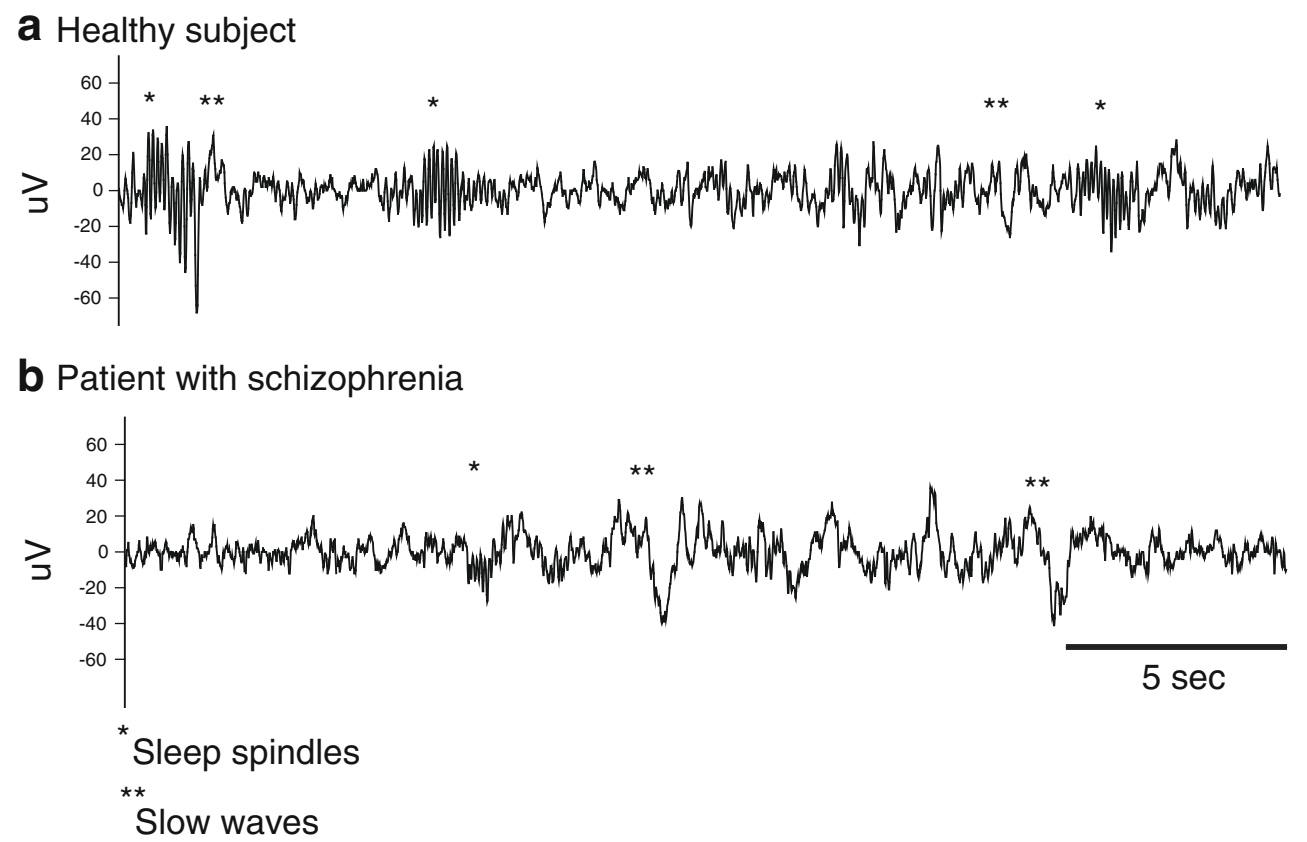


the lateral geniculate nucleus (LGN) and reduced firing of TRN [33], whereas pharmacologically induced decrease in TRN firings resulted in auditory gating deficits [34]. Of note, sensory gating and attention deficits have been reported in several groups of schizophrenia patients [35], and are considered part of the core feature of this disorder. In addition to the TRN, recent evidence suggests that the mediodorsal (MD) nucleus may also be implicated in sleep spindle deficits. The $\mathrm{MD}$ is a higher order (HO) nucleus, which receives driver inputs from cortical layer $\mathrm{V}$ neurons, whereas first-order (FO) thalamic relays, such as the lateral geniculate nuclei (LGN), are projected to primarily from subcortical sources [36], and in a recent study, we found that the MD nuclei, but not the LGN, were significantly smaller in schizophrenia patients compared to healthy controls [37॰]. Additionally, left MD volumes were correlated with left frontal EEG spindles in both healthy and schizophrenic subjects, a finding consistent with electrocorticogram recordings in humans showing an implication of MD and TRN in sleep spindles occurring in the prefrontal cortex [38].

Abnormalities in cortico-thalamic projections could also play a role in sleep spindle deficits in schizophrenia. Converging evidence from neuroimaging [39], electrophysiological [40], as well as postmortem [41] studies have revealed cortico-thalamic connectivity deficits in patients with schizophrenia. Furthermore, cortical neurons have been shown to contribute to the initiation and termination of spindle activity. A recent study combining local field potential in the medial prefrontal cortex and single-unit recordings in the TRN in freely behaving rats identified "early" cortical cells that fired in synchrony with TRN spikes as well as "late" neurons that fired in antiphase to reticular thalamic neurons. In interpreting these findings, the authors suggested that early cortical cells contribute to initiate spindle activity in the cortex, whereas late neurons contribute to the termination of the spindle oscillation [42]. Altogether, these findings support the presence of TRNMD thalamus-prefrontal circuit deficits in schizophrenia.

The molecular mechanisms underlying sleep spindle impairments in schizophrenia have yet to be elucidated. However, abnormalities in $\mathrm{Ca}+$ channel activity are likely to play a significant role. Electrophysiological recordings in primates have shown that MD nucleus has higher burst firing compared to the LGN [43], and this burst propensity is due to a greater expression of voltage-dependent, transient (T-type) calcium channels [44]. TRN neurons also have high intracellular $\mathrm{Ca} 2+$ to sustain the rhythmic bursting necessary for spindle generation, which involves low-voltage T-type $\mathrm{Ca} 2+$ channels [45•]. During NREM sleep, a progressive hyperpolarization of TRN neurons favors the activation of $\mathrm{T}$ channels that elicit rapid, transient bursts of action potentials [46]. Reticular thalamic neurons express two T channel subtypes encoded by the CaV3.2 (CACNA1h) and the CaV3.3 CACNA1i) genes [47], and genetic deletion of $\mathrm{CaV} 3.3$ channels markedly reduces $\mathrm{T}$ currents, thus preventing the low-threshold bursting necessary to sustain spindle oscillations [48•]. Intriguingly CACNA1I, a gene encoding a T-type calcium channel, $\mathrm{CaV} 3.3$, has been recently shown to be strongly associated to schizophrenia by two large genetic studies $[49,50 \bullet]$, and another study has demonstrated that the CaV3.3 calcium channel, which is highly expressed in the TRN, is the major sleep spindle pacemaker in the thalamus [48•].

Another potential molecular mechanism underlying spindle deficits involves abnormalities in GABA-ergic neurotransmission. Electrophysiological recordings in rodents have recently shown that, during development, GABA currents depolarize TRN neurons and regulate the bursting activity observed during sleep spindles [51]. TRN GABA release plays also a critical role in regulating auditory sensory gating [34], which has been consistently found to be defective in schizophrenia [52]. Moreover, postmortem studies have reported a reduction in glutamate decarboxylase 67, an enzyme involved in GABA synthesis, and in GABA membrane transporter density in cortical interneurons in schizophrenia patients [53]. Treatment studies have shown that clozapine, the most effective antipsychotic currently available in schizophrenia, is associated with enhanced thalamocortical GABA activity, whereas the beneficial effects of electro-convulsive therapy (ECT) and transcranial magnetic stimulation (TMS) appears to be related to an increased GABA neurotransmission on excitatory cortical neurons [54].

One additional molecular mechanism which could be implicated in sleep spindle deficits is a reduced N-methyl-D-aspartate (NMDA) glutamate receptor activity in the thalamocortical system. Beside the TRN, which consists entirely of GABA-ergic inhibitory neurons, most neurons in the thalamus and in the cortex are glutamatergic and converging evidence indicates a reduced binding or expression of thalamo-cortical NMDA receptors in schizophrenia. Postmortem studies found reduced NMDA receptors in both the MD thalamus and the prefrontal cortex of schizophrenia patients [55]. Pharmacological manipulations with NMDA antagonists, such as ketamine and phencyclidine (PCP), induces schizophrenia-like psychosis in healthy individuals, and animal studies have shown that asenapine and clozapine, two second-generation antipsychotics, could revert a PCP-induced hypoactivity of NMDA receptors in both MD thalamus [56] and prefrontal cortex [57]. Injections of NMDA antagonists in the TRN rat brain trigger delta-range rhythmic bursting, thus suggesting that NMDA hypofunction underlie TRN-generated delta band EEG oscillations, a waking thalamo-cortical dysrhythmia established in schizophrenia [58]. Furthermore, 2deoxyglucose imaging data in mice characterizing the acute effects of ketamine on brain functional connectivity found ketamine-induced impairments in a circuitry involving TRN, MD thalamus, and prefrontal cortex [59]. 


\section{Conclusions}

Over the past several years, there has been a renewed interest in investigating psychiatric disorders, and especially schizophrenia, during sleep [60]. Sleep represents the ultimate resting state, and sleep specific brain rhythms reflect the activity of complementary circuits within the thalamo-cortical system. Furthermore, the recent availability of high density EEG systems has made it possible to combine the exquisite temporal resolution provided by traditional electroencephalographic recordings with good spatial characterization of neuronal oscillations [61]. Capitalizing on this approach, several recent studies have reported marked deficits in several sleep spindles parameters in patients with schizophrenia compared to both healthy subjects and other psychiatric patients. These deficits were correlated with some of the symptoms commonly experienced in patients with schizophrenia and seemed to point to a dysfunction in a TRN-MD thalamus-prefrontal cortex circuit $[25 \bullet, 26 \bullet, 37 \bullet$, ]. Additionally, spindle impairments were recently established in early course schizophrenia patients as well as first-degree relatives of schizophrenia probands, and spindle measures correlated with impaired executive functions, positive symptoms, and IQ estimates [30•].

Future work is needed to fully establish the extent of sleep spindle deficits in schizophrenia, including multi-site studies in large group of patients. One important aspect to clarify will be the specificity of spindle abnormalities as a sleep finding in patients with schizophrenia. In a recent study, we found no difference in several parameters, including incidence, negative peak amplitude, down-slopes, as well as up-slopes of sleep slow waves in patients with schizophrenia compared to healthy and psychiatric controls. Nonetheless, source modeling analysis of slow wave activity could reveal local deficits in cortical areas (i.e., dorsolateral prefrontal cortex), which have consistently found to be impaired in patients with schizophrenia. Furthermore, given the increasing evidence of the role of slow wave activity (SWA) in memory consolidation and learning in healthy humans $[62,63]$, future studies should investigate whether an abnormal modulation in SWA, especially following cognitive tasks that show a performance improvement after sleep, may predict some of the cognitive deficits commonly observed in patients with schizophrenia, and may help reveal the neuronal circuits underlying these impaired performances. It will also be critical to assess whether abnormalities in spindle activity can be observed during the prodromal phase in individuals at high risk of conversion to psychosis, and whether these abnormalities can predict illness onset. Finally, the effects of pharmacological as well as nonpharmacological interventions on sleep spindle deficits and their impact on the course of illness should be investigated in patients with schizophrenia. For example, in a pharmacological study, it was found that eszopiclone, an hypnotic medication that activates GABAergic neurons, significantly increased sleep spindles in patients with schizophrenia, and the spindle increase correlated with improvements in an overnight motor sequence task when placebo and eszopiclone groups were combined for analysis [12•]. Additionally, by employing TMS during NREM sleep, it was recently demonstrated that both slow waves and sleep spindles can be evoked in healthy subjects [64], whereas it has been suggested that sleep spindles can be induced by transcranial electrical stimulation (TES) during sleep in schizophrenia patients, which should help supplement their low spindle incidence [65].

\section{Compliance with Ethics Guidelines}

Conflict of Interest Fabio Ferrarelli declares that he has no conflict of interest.

Human and Animal Rights and Informed Consent This article does not contain any studies with human or animal subjects performed by any of the authors.

\section{References}

Papers of particular interest, published recently, have been highlighted as:

- Of importance

1. Whiteford HA, Degenhardt L, Rehm J, Baxter AJ, Ferrari AJ, Erskine HE, et al. Global burden of disease attributable to mental and substance use disorders: findings from the Global Burden of Disease Study 2010. Lancet. 2013;382(9904):1575-86.

2. Fusar-Poli P, Politi P. Paul Eugen Bleuler and the birth of schizophrenia (1908). Am J Psychiatry. 2008;165(11):1407.

3. Barch DM, Bustillo J, Gaebel W, Gur R, Heckers S, Malaspina D, et al. Logic and justification for dimensional assessment of symptoms and related clinical phenomena in psychosis: relevance to DSM-5. Schizophr Res. 2013;150(1):15-20.

4. Gur RE, Calkins ME, Gur RC, Horan WP, Nuechterlein KH, Seidman LJ, et al. The Consortium on the Genetics of Schizophrenia: neurocognitive endophenotypes. Schizophr Bull. 2007;33(1):49-68.

5. Onitsuka T, Oribe N, Nakamura I, Kanba S. Review of neurophysiological findings in patients with schizophrenia. Psychiatry Clin Neurosci. 2013;67(7):461-70.

6. Schaefer J, Giangrande E, Weinberger DR, Dickinson D. The global cognitive impairment in schizophrenia: consistent over decades and around the world. Schizophr Res. 2013;150(1):42-50.

7. Riedner BA, Hulse BK, Murphy MJ, Ferrarelli F, Tononi G. Temporal dynamics of cortical sources underlying spontaneous and peripherally evoked slow waves. Prog Brain Res. 2011;193: 201-18.

8. Luthi A. Sleep spindles: where they come from, what they do. Neurosci Rev J Bring Neurobiol Neurol Psychiatry. 2013;20(3): 243-56. doi:10.1177/1073858413500854.

9. Diekelmann S, Born J. The memory function of sleep. Nat Rev Neurosci. 2010;11(2):114-26. 
10. Tononi G. Slow wave homeostasis and synaptic plasticity. J Clin Sleep Med JCSM Off Publ Am Acad Sleep Med. 2009;5(2 Suppl): S16-9.

11. Manoach DS, Stickgold R. Does abnormal sleep impair memory consolidation in schizophrenia? Front Hum Neurosci. 2009;3:21.

12. Wamsley EJ, Shinn AK, Tucker MA, Ono KE, McKinley SK, Ely AV, et al. The effects of eszopiclone on sleep spindles and memory consolidation in schizophrenia: a randomized placebo-controlled trial. Sleep. 2013;36(9):1369-76. This is the first study showing a pharmacologically mediated improvement in sleep spindle incidence in patients with schizophrenia.

13. Monti JM, Monti D. Sleep disturbance in schizophrenia. Int Rev Psychiatry. 2005;17(4):247-53.

14. Kahn-Greene ET, Killgore DB, Kamimori GH, Balkin TJ, Killgore WD. The effects of sleep deprivation on symptoms of psychopathology in healthy adults. Sleep Med. 2007;8(3):215-21.

15. Miller TJ, Zipursky RB, Perkins D, Addington J, Woods SW, Hawkins KA, et al. The PRIME North America randomized double-blind clinical trial of olanzapine versus placebo in patients at risk of being prodromally symptomatic for psychosis. II. Baseline characteristics of the "prodromal" sample. Schizophr Res. 2003;61(1):19-30.

16. Gordon JA, Moore H. Charting a course toward an understanding of schizophrenia. Neuron. 2012;76(3):465-7.

17. Keefe RS. The longitudinal course of cognitive impairment in schizophrenia: an examination of data from premorbid through posttreatment phases of illness. J Clin Psychiatry. 2014;75 Suppl 2:8-13.

18. Yang C, Winkelman JW. Clinical significance of sleep EEG abnormalities in chronic schizophrenia. Schizophr Res. 2006;82(2-3): 251-60.

19. Poulin J, Daoust AM, Forest G, Stip E, Godbout R. Sleep architecture and its clinical correlates in first episode and neuroleptic-naive patients with schizophrenia. Schizophr Res. 2003;62(1-2):147-53.

20. Keshavan MS, Reynolds 3rd CF, Miewald MJ, Montrose DM, Sweeney JA, Vasko Jr RC, et al. Delta sleep deficits in schizophrenia: evidence from automated analyses of sleep data. Arch Gen Psychiatry. 1998;55(5):443-8.

21. Chouinard S, Poulin J, Stip E, Godbout R. Sleep in untreated patients with schizophrenia: a meta-analysis. Schizophr Bull. 2004;30(4):957-67.

22. Hiatt JF, Floyd TC, Katz PH, Feinberg I. Further evidence of abnormal non-rapid-eye-movement sleep in schizophrenia. Arch Gen Psychiatry. 1985;42(8):797-802.

23. Goder R, Aldenhoff JB, Boigs M, Braun S, Koch J, Fritzer G. Delta power in sleep in relation to neuropsychological performance in healthy subjects and schizophrenia patients. J Neuropsychiatry Clin Neurosci. 2006;18(4):529-35.

24. Tekell JL, Hoffmann R, Hendrickse W, Greene RW, Rush AJ, Armitage R. High frequency EEG activity during sleep: characteristics in schizophrenia and depression. Clin EEG Neurosci. 2005;36(1):25-35.

25. Ferrarelli F, Huber R, Peterson MJ, Massimini M, Murphy M, Riedner BA, et al. Reduced sleep spindle activity in schizophrenia patients. Am J Psychiatry. 2007;164(3):483-92. This is the first study showing spindle activity deficits in patients with schizophrenia employing hd-EEG.

26. Ferrarelli F, Peterson MJ, Sarasso S, Riedner BA, Murphy MJ, Benca RM, et al. Thalamic dysfunction in schizophrenia suggested by whole-night deficits in slow and fast spindles. Am J Psychiatry. 2010;167(11):1339-48. In this follow-up study it was shown that spindle deficits were unrelated to antipsychotic medications and they were likely specific for schizophrenia patients.

27. Boutros NN, Mucci A, Vignapiano A, Galderisi S Electrophysiological aberrations associated with negative symptoms in schizophrenia. Curr Top Behav Neurosci. 2014. doi:10.1007/7854 2014303.

28. Van Cauter E, Linkowski P, Kerkhofs M, Hubain P, L'HermiteBaleriaux M, Leclercq R, et al. Circadian and sleep-related endocrine rhythms in schizophrenia. Arch Gen Psychiatry. 1991;48(4): $348-56$.

29. Ferrarelli F, Tononi G. The thalamic reticular nucleus and schizophrenia. Schizophr Bull. 2011;37(2):306-15.

30. Manoach DS, Demanuele C, Wamsley EJ, Vangel M, Montrose DM, Miewald J, et al. Sleep spindle deficits in antipsychoticnaive early course schizophrenia and in non-psychotic first-degree relatives. Front Hum Neurosci. 2014;8:762. This study established reduced spindle activity in first-break schizophrenia patients and their relatives compared to healthy controls and to early course patients with other psychotic disorders.

31. Pinault D. The thalamic reticular nucleus: structure, function and concept. Brain Res Brain Res Rev. 2004;46(1):1-31.

32. Fuentealba P, Steriade M. The reticular nucleus revisited: intrinsic and network properties of a thalamic pacemaker. Prog Neurobiol. 2005;75(2):125-41.

33. McAlonan K, Cavanaugh J, Wurtz RH. Guarding the gateway to cortex with attention in visual thalamus. Nature. 2008;456(7220): $391-4$

34. Krause M, Hoffmann WE, Hajos M. Auditory sensory gating in hippocampus and reticular thalamic neurons in anesthetized rats. Biol Psychiatry. 2003;53(3):244-53.

35. Freedman R, Ross R, Leonard S, Myles-Worsley M, Adams CE, Waldo M, et al. Early biomarkers of psychosis. Dialogues Clin Neurosci. 2005;7(1):17-29.

36. Sherman SM, Guillery RW. The role of the thalamus in the flow of information to the cortex. Philos Trans R Soc Lond B Biol Sci. 2002;357(1428):1695-708.

37. Buchmann A, Dentico D, Peterson MJ, Riedner BA, Sarasso S, Massimini M, et al. Reduced mediodorsal thalamic volume and prefrontal cortical spindle activity in schizophrenia. Neuroimage. 2014;102(Pt 2):540-7. This study's findings point to a TRNMD thalamus-prefrontal cortex circuit defect, which may underie spindle deficits and cognitive impairment in schizophrenia.

38. Nakamura M, Uchida S, Maehara T, Kawai K, Hirai N, Nakabayashi T, et al. Sleep spindles in human prefrontal cortex: an electrocorticographic study. Neurosci Res. 2003;45(4):419-27.

39. Camchong J, Dyckman KA, Chapman CE, Yanasak NE, McDowell JE. Basal ganglia-thalamocortical circuitry disruptions in schizophrenia during delayed response tasks. Biol Psychiatry. 2006;60(3):235-41.

40. Ferrarelli F, Massimini M, Peterson MJ, Riedner BA, Lazar M, Murphy MJ, et al. Reduced evoked gamma oscillations in the frontal cortex in schizophrenia patients: a TMS/EEG study. Am J Psychiatry. 2008;165(8):996-1005.

41. Akbarian S, Kim JJ, Potkin SG, Hetrick WP, Bunney Jr WE, Jones EG. Maldistribution of interstitial neurons in prefrontal white matter of the brains of schizophrenic patients. Arch Gen Psychiatry. 1996;53(5):425-36.

42. Gardner RJ, Hughes SW, Jones MW. Differential spike timing and phase dynamics of reticular thalamic and prefrontal cortical neuronal populations during sleep spindles. J Neurosci. 2013;33(47): 18469-80.

43. Ramcharan EJ, Gnadt JW, Sherman SM. Higher-order thalamic relays burst more than first-order relays. Proc Natl Acad Sci U S A. 2005;102(34):12236-41.

44. Wei H, Bonjean M, Petry HM, Sejnowski TJ, Bickford ME. Thalamic burst firing propensity: a comparison of the dorsal lateral geniculate and pulvinar nuclei in the tree shrew. J Neurosci. 2011;31(47):17287-99. 
45. Astori S, Wimmer RD, Luthi A. Manipulating sleep spindlesexpanding views on sleep, memory, and disease. Trends Neurosci. 2013;36(12):738-48. This is an elegant review on the current understanding of spindles in sleep, memory, and mental illness.

46. Huguenard JR. Low-threshold calcium currents in central nervous system neurons. Annu Rev Physiol. 1996;58:329-48. doi:10.1146/ annurev.ph.58.030196.001553.

47. Talley EM, Cribbs LL, Lee JH, Daud A, Perez-Reyes E, Bayliss DA. Differential distribution of three members of a gene family encoding low voltage-activated (T-type) calcium channels. J Neurosci Off J Soc Neurosci. 1999;19(6):1895-911.

48. Astori S, Wimmer RD, Prosser HM, Corti C, Corsi M, Liaudet N, et al. The $\mathrm{Ca}(\mathrm{V}) 3.3$ calcium channel is the major sleep spindle pacemaker in thalamus. Proc Natl Acad Sci U S A. 2011;108(33): 13823-8. This study shows that the $\mathbf{C a}(\mathbf{V}) 3.3$ calcium channel, which is expressed abundantly in the TRN, is the major sleep spindle pacemaker in thalamus.

49. Irish Schizophrenia Genomics $\mathrm{C}$, the Wellcome Trust Case Control C. Genome-wide association study implicates HLA-C*01:02 as a risk factor at the major histocompatibility complex locus in schizophrenia. Biol Psychiatry. 2012;72(8):620-8.

50. Schizophrenia Working Group of the Psychiatric Genomics C. Biological insights from 108 schizophrenia-associated genetic loci. Nature. 2014;511(7510):421-7. This large multi-stage schizophrenia genome-wide association studypresents 108 loci, including genes regulating calcium channels, significantly associated to schizophrenia.

51. Pangratz-Fuehrer S, Rudolph U, Huguenard JR. Giant spontaneous depolarizing potentials in the developing thalamic reticular nucleus. J Neurophysiol. 2007;97(3):2364-72.

52. Cadenhead KS, Light GA, Shafer KM, Braff DL. P50 suppression in individuals at risk for schizophrenia: the convergence of clinical, familial, and vulnerability marker risk assessment. Biol Psychiatry. 2005;57(12):1504-9.

53. Lewis DA, Hashimoto T, Volk DW. Cortical inhibitory neurons and schizophrenia. Nat Rev Neurosci. 2005;6(4):312-24.

54. Daskalakis ZJ, Christensen BK, Fitzgerald PB, Moller B, Fountain SI, Chen R. Increased cortical inhibition in persons with schizophrenia treated with clozapine. J Psychopharmacol. 2008;22(2):203-9.

55. Pakkenberg B, Scheel-Kruger J, Kristiansen LV. Schizophrenia; from structure to function with special focus on the mediodorsal thalamic prefrontal loop. Acta Psychiatr Scand. 2009;120(5):34554.

56. Santana N, Troyano-Rodriguez E, Mengod G, Celada P, Artigas F. Activation of thalamocortical networks by the N-methyl-Daspartate receptor antagonist phencyclidine: reversal by clozapine. Biol Psychiatry. 2011;69(10):918-27.

57. Jardemark K, Marcus MM, Shahid M, Svensson TH. Effects of asenapine on prefrontal N-methyl-D-aspartate receptor-mediated transmission: involvement of dopamine D1 receptors. Synapse. 2010;64(11):870-4.

58. Zhang Y, Llinas RR, Lisman JE. Inhibition of NMDARs in the nucleus reticularis of the thalamus produces delta frequency bursting. Front Neural Circ. 2009;3:20.

59. Dawson N, Morris BJ, Pratt JA. Subanaesthetic ketamine treatment alters prefrontal cortex connectivity with thalamus and ascending subcortical systems. Schizophr Bull. 2013;39(2):366-77.

60. Wilson S, Argyropoulos S. Sleep in schizophrenia: time for closer attention. Br J Psychiatry. 2012;200(4):273-4.

61. Pisarenco I, Caporro M, Prosperetti C, Manconi M. High-density electroencephalography as an innovative tool to explore sleep physiology and sleep related disorders. Int J Psychophysiol. 2014.

62. Born J, Wilhelm I. System consolidation of memory during sleep. Psychol Res. 2012;76(2):192-203.

63. Massimini M, Tononi G, Huber R. Slow waves, synaptic plasticity and information processing: insights from transcranial magnetic stimulation and high-density EEG experiments. Eur J Neurosci. 2009;29(9):1761-70.

64. Massimini M, Ferrarelli F, Esser SK, Riedner BA, Huber R, Murphy M, et al. Triggering sleep slow waves by transcranial magnetic stimulation. Proc Natl Acad Sci U S A. 2007;104(20):8496501.

65. Buzsaki G, Watson BO. Brain rhythms and neural syntax: implications for efficient coding of cognitive content and neuropsychiatric disease. Dialogues Clin Neurosci. 2012;14(4):345-67. 